OCU-PHYS 319

AP-GR 71

\title{
Geodetic Precession
}

\section{in Squashed Kaluza-Klein Black Hole Spacetimes}

\author{
Ken Matsuno* and Hideki Ishihara ${ }^{\dagger}$ \\ Department of Mathematics and Physics, \\ Graduate School of Science, Osaka City University, \\ 3-3-138 Sugimoto, Sumiyoshi-ku, Osaka 558-8585, Japan
}

(Dated: December 1, 2018)

\begin{abstract}
We investigate the geodetic precession effect of a parallely transported spin-vector along a circular geodesic in the five-dimensional squashed Kaluza-Klein black hole spacetime. Then we derive the higher-dimensional correction of the precession angle to the general relativity. We find that the correction is proportional to the square of (size of extra dimension)/(gravitational radius of central object).

PACS numbers: 04.50.-h, 04.70.Bw
\end{abstract}

\footnotetext{
*E-mail: matsuno@sci.osaka-cu.ac.jp

$\dagger$ E-mail: ishihara@sci.osaka-cu.ac.jp
} 


\section{INTRODUCTION}

Recently, classical general relativity in higher dimensions, suggested by superstring theory, has gathered much attention. Especially, many studies are devoted to higherdimensional black holes (see [1] for review) because black holes would be expected as windows to extra dimensions. One of the most interesting problems is a verification of extra dimensions by physical phenomena related to the higher-dimensional black holes. Higher-

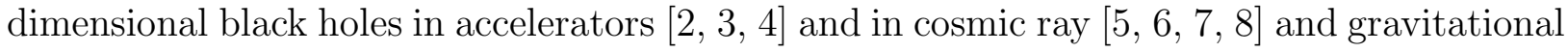
waves from higher-dimensional black holes [9] are studied for this purpose.

In astrophysics, the study of the classical tests of the general relativity: the light deflection, the perihelion shift, the time delay, could provide methods to know properties of the spacetime metrics around compact objects. In addition, the precession of a gyroscope [10, 11, 12] is another experimental way to reveal the spacetime structure. The studies of these effects open the possibility of testing higher-dimensional models by using astronomical and astrophysical observations at the solar system scale. Indeed, such problems are considered by using five-dimensional two-parameter family of Kaluza-Klein type solutions with an $S^{2}$ symmetry [13, 14, 15, 16, 17, 18], and $S^{2}$ symmetric solutions in brane world models [19, 20, 21, 22].

Note that the exterior spacetime is described by the Schwarzschild metric for standard general relativistic spherical compact objects. However, in higher-dimensional spacetime models, the exterior metric of a static star is no longer the Schwarzschild metric. In this paper, we consider another class of Kaluza-Klein type metrics which would describe exterior of compact objects. In the higher-dimensional spacetimes, even if we impose the asymptotic flatness to the four-dimensional part of the spacetime, there are various possibilities of fiber bundle structures of the extra dimensions as the fiber over the four-dimensional base spacetime. The black hole solutions with non-trivial bundle structures have been studied by various authors. Dobiasch and Maison found the first five-dimensional vacuum Kaluza-Klein black hole solution which asymptotes to a twisted $\mathrm{S}^{1}$ fiber bundle over the four-dimensional Minkowski spacetime [23], and Gibbons and Wiltshire clarified the meaning of the metric [24]. Later, the solution was generalized to the rotating case [25], and charged case in the five-dimensional Einstein-Maxwell theory [26]. It is shown that this family of black holes have squashed $\mathrm{S}^{3}$ horizons, and that the metrics behave as fully five-dimensional black 
holes in the vicinity of horizon, while behave as four-dimensional black holes in the far region. The squashed Kaluza-Klein black hole solutions of cohomogeneity-one are related to asymptotically flat black hole solutions by a squashing function [26, 27]. Some of the supersymmetric black hole solutions [28, 29, 30] in the framework of ref. [31] and nonsupersymmetric solutions [32, 33, 34, 35, 36, 37] were constructed by the procedure of squashing. These solutions also have been generalized in various directions, e.g., in EinsteinYang-Mills theory [38], and in Einstein-Maxwell-dilaton theory [39, 40].

Motivated by the Gravity Probe B experiment [12], we focus our attention to the precession of a gyroscope in an orbit around the Earth. We assume that the geometry of the region outside the Earth is described by the five-dimensional vacuum static squashed Kaluza-Klein black hole metric [23, 26]. We derive the higher-dimensional correction of the precession angle to the general relativity, which is related with the size of the extra dimension.

We regard a rotating axis of the gyroscope carried by a satellite as a spacelike spin-vector. While the satellite moves along the stable circular orbit around the Earth, the spin-vector is parallely transported along this timelike circular geodesic. When the satellite returns to the initial position, an angular difference of the final spin-vector from the initial one appears. This is the geodetic effect [10]. Since the Earth rotates, the spin-vector is dragged around the rotation axis of the Earth by the Kerr geometry [11]. The frame dragging effect by the Kerr rotation is quite smaller than the geodetic effect, then, we investigate the correction by the extra dimensions to the geodetic effect in this paper.

This paper is organized as follows. In the next section, we review the properties of fivedimensional static squashed Kaluza-Klein black hole solution. In section III, we show that the five-dimensional squashed Kaluza-Klein black hole spacetime admits stable circular orbits similar to the four-dimensional Schwarzschild black holes. In section IV, we consider the parallel transportation of a spin-vector along a circular orbit in the squashed Kaluza-Klein black hole spacetime, then derive the geodetic precession angle with the higher-dimensional correction. Finally, we devote section $\mathrm{V}$ to discussion.

\section{REVIEW OF SQUASHED KALUZA-KLEIN BLACK HOLES}

We start from the five-dimensional vacuum Einstein equation,

$$
R_{\mu \nu}=0
$$


The static Kaluza-Klein black hole with a squashed $S^{3}$ horizon [23, 24] is one of the exact solutions of (1). The metric is written as [26]

$$
\begin{aligned}
& d s^{2}=-V(\rho) d t^{2}+U(\rho)\left[\frac{d \rho^{2}}{V(\rho)}+\rho^{2} d \Omega_{S^{2}}^{2}\right]+\frac{r_{\infty}^{2}}{4 U(\rho)}(d \psi+\cos \theta d \phi)^{2} \\
& d \Omega_{S^{2}}^{2}:=d \theta^{2}+\sin ^{2} \theta d \phi^{2}
\end{aligned}
$$

where the functions $V(\rho), U(\rho)$ are given by

$$
V(\rho)=1-\frac{\rho_{g}}{\rho}, \quad U(\rho)=1+\frac{\rho_{0}}{\rho}
$$

and positive parameters $r_{\infty}, \rho_{g}$ and $\rho_{0}$ are related as $r_{\infty}^{2}=4 \rho_{0}\left(\rho_{g}+\rho_{0}\right)$. The ranges of coordinates are $-\infty<t<\infty, 0<\rho<\infty, 0 \leq \theta \leq \pi, 0 \leq \phi \leq 2 \pi$, and $0 \leq \psi \leq 4 \pi$.

The black hole horizon is located at $\rho=\rho_{g}$, and the curvature singularity at $\rho=0$ is concealed behind it. The induced metric on the three-dimensional cross section of the black hole horizon with a time slice $t=$ const. is obtained as

$$
\left.d s^{2}\right|_{\rho=\rho_{g}, t=\text { const. }}=\rho_{g} \rho_{0}\left[\left(1+\frac{\rho_{g}}{\rho_{0}}\right) d \Omega_{S^{2}}^{2}+(d \psi+\cos \theta d \phi)^{2}\right] .
$$

Then, we see that the metric of black hole horizon is the squashed $S^{3}$ in the form of the Hopf bundle. Since $\rho_{g}>0$ and $\rho_{0}>0$, we see that the radius of the $\mathrm{S}^{2}$ base is larger than that of the $\mathrm{S}^{1}$ fiber.

At the infinity, $\rho=\infty$, the metric (2) behaves as

$$
d s^{2} \simeq-d t^{2}+d \rho^{2}+\rho^{2} d \Omega_{S^{2}}^{2}+\frac{r_{\infty}^{2}}{4}(d \psi+\cos \theta d \phi)^{2} .
$$

That is, the metric (2) asymptotes to a twisted constant $\mathrm{S}^{1}$ fiber bundle over the fourdimensional Minkowski spacetime. The size of compactified extra dimension of the spacetime (2) at the infinity is given by $r_{\infty}$. The Komar mass ${ }^{1}$ of this black hole (2) is given by

$$
M=\frac{\pi r_{\infty} \rho_{g}}{G_{5}}=\frac{\rho_{g}}{2 G_{4}}
$$

where the five-dimensional gravitational constant $G_{5}$ and the four-dimensional one $G_{4}$ are related as

$$
G_{4}=\frac{G_{5}}{2 \pi r_{\infty}}
$$

\footnotetext{
1 For the squashed black hole, the Komar mass takes a different value of the Abbott-Deser mass [41, 42].
} 
Here, we discuss the physical meanings of the parameter $\rho_{0}$ in (2). If $\rho_{0} \ll \rho_{g}$, $\rho$ dependence of the function $V(\rho)$ is important but the function $U(\rho)$ is almost unity for an observer outside the horizon, $\rho_{0} \ll \rho_{g} \lesssim \rho$. Then, the observer feels the spacetime as the four-dimensional Schwarzschild black hole with a constant twisted $\mathrm{S}^{1}$ fiber with the metric

$$
d s^{2} \simeq-\left(1-\frac{\rho_{g}}{\rho}\right) d t^{2}+\left(1-\frac{\rho_{g}}{\rho}\right)^{-1} d \rho^{2}+\rho^{2} d \Omega_{S^{2}}^{2}+\frac{r_{\infty}^{2}}{4}(d \psi+\cos \theta d \phi)^{2} .
$$

On the other hand, if $\rho_{g} \ll \rho_{0}$, the function $U(\rho)$ becomes important for an observer at $\rho_{g} \lesssim \rho \ll \rho_{0}$. With the help of a new coordinate $r=2 \sqrt{\rho_{0} \rho}$ and a parameter $r_{g}=2 \sqrt{\rho_{0} \rho_{g}}$, since $r_{g}^{2} \ll r_{\propto}^{2}$, the metric (2) approaches to the five-dimensional Schwarzschild-Tangherlini black hole [43]:

$$
d s^{2} \simeq d s_{\mathrm{ST}}^{2}=-\left(1-\frac{r_{g}^{2}}{r^{2}}\right) d t^{2}+\left(1-\frac{r_{g}^{2}}{r^{2}}\right)^{-1} d r^{2}+r^{2} d \Omega_{S^{3}}^{2},
$$

where $d \Omega_{S^{3}}^{2}$ denotes the metric of the unit three-sphere. Then, the observer feels the spacetime as an almost $S^{3}$ symmetric black hole. Therefore, the parameter $\rho_{0}$ gives the typical scale of transition from five-dimension to effective four-dimension.

\section{CIRCULAR ORBITS AROUND SQUASHED KALUZA-KLEIN BLACK HOLES}

We consider timelike geodesics in the five-dimensional squashed Kaluza-Klein black hole. The Lagrangian for a test particle in the metric (2) is

$$
\mathcal{L}=\frac{1}{2}\left[-V \dot{t}^{2}+\frac{U}{V} \dot{\rho}^{2}+U \rho^{2}\left(\dot{\theta}^{2}+\sin ^{2} \theta \dot{\phi}^{2}\right)+\frac{r_{\infty}^{2}}{4 U}(\dot{\psi}+\cos \theta \dot{\phi})^{2}\right],
$$

where the overdot denotes the differentiation with respect to the proper time $\tau$, then we set the condition $2 \mathcal{L}=-1$. We can obtain three constants of motion,

$$
\begin{aligned}
E & :=V \dot{t} \\
L & :=U \rho^{2} \sin ^{2} \theta \dot{\phi}+\frac{r_{\infty}^{2} \cos \theta}{4 U}(\dot{\psi}+\cos \theta \dot{\phi}), \\
p_{\psi} & :=\frac{r_{\infty}^{2}}{4 U}(\dot{\psi}+\cos \theta \dot{\phi}) .
\end{aligned}
$$

Here, we assume that the particle has no momentum in the extra direction, i.e., $p_{\psi}=0$. The effective Lagrangian for the particle is

$$
\mathcal{L}_{\text {eff }}=\frac{1}{2}\left[-V \dot{t}^{2}+\frac{U}{V} \dot{\rho}^{2}+U \rho^{2}\left(\dot{\theta}^{2}+\sin ^{2} \theta \dot{\phi}^{2}\right)\right],
$$


where $2 \mathcal{L}_{\text {eff }}=-1$. We see that this effective Lagrangian has the same form in the case of four-dimensional spherically symmetric spacetimes. Then, we can concentrate on orbits with $\theta=\pi / 2$ on the assumption of $p_{\psi}=0$. In these conditions, we obtain the energy conservation equation

$$
\left(1+\frac{\rho_{0}}{\rho}\right)\left(\frac{d \rho}{d \tau}\right)^{2}+V_{\mathrm{KK}}(\rho)=E^{2},
$$

where the effective potential is given by

$$
V_{\mathrm{KK}}(\rho)=\left(1-\frac{\rho_{g}}{\rho}\right)\left(1+\frac{L^{2}}{\rho\left(\rho+\rho_{0}\right)}\right) .
$$

We note that (16) with (17) reduces to the energy conservation equation of a particle in the four-dimensional Schwarzschild black hole spacetime in the limit $\rho_{0} \rightarrow 0$. A typical shape of the effective potential $V_{\mathrm{KK}}$ is shown in the left panel of Figure 1. We see that there is a stable circular orbit at the local minimum of the effective potential.

The squashed Kaluza-Klein black holes, which have stable circular orbits, make remarkable contrast with the higher-dimensional asymptotically flat black holes, which have no stable bound state of particles. For the simplest example, we consider timelike geodesics in the five-dimensional Schwarzschild-Tangherlini black hole spacetime with the metric (10). We consider motions in an equatorial plane, that is, $\theta=\pi / 2$, then we have the energy conservation equation

$$
\left(\frac{d r}{d \tau}\right)^{2}+V_{\mathrm{ST}}(r)=E_{\mathrm{ST}}^{2}
$$

with the effective potential

$$
V_{\mathrm{ST}}(r)=\left(1-\frac{r_{g}^{2}}{r^{2}}\right)\left(1+\frac{L_{\mathrm{ST}}^{2}}{r^{2}}\right)
$$

where $E_{\mathrm{ST}}$ and $L_{\mathrm{ST}}$ are constants of motion of the system [44]. A typical shape of the effective potential is drawn in the right panel of Figure 1. Clearly, there is no stable circular orbit. The metric of squashed Kaluza-Klein black hole (2) with a condition $\rho_{0} \ll \rho_{g}$ can be a candidate for the metric which describes a spacetime around the Earth where a test particle can travel along a circular orbit. We can expect the appearance of the higher-dimensional correction, which is related to the parameter $\rho_{0}$, to the geodetic precession effect of fourdimensional relativity. 

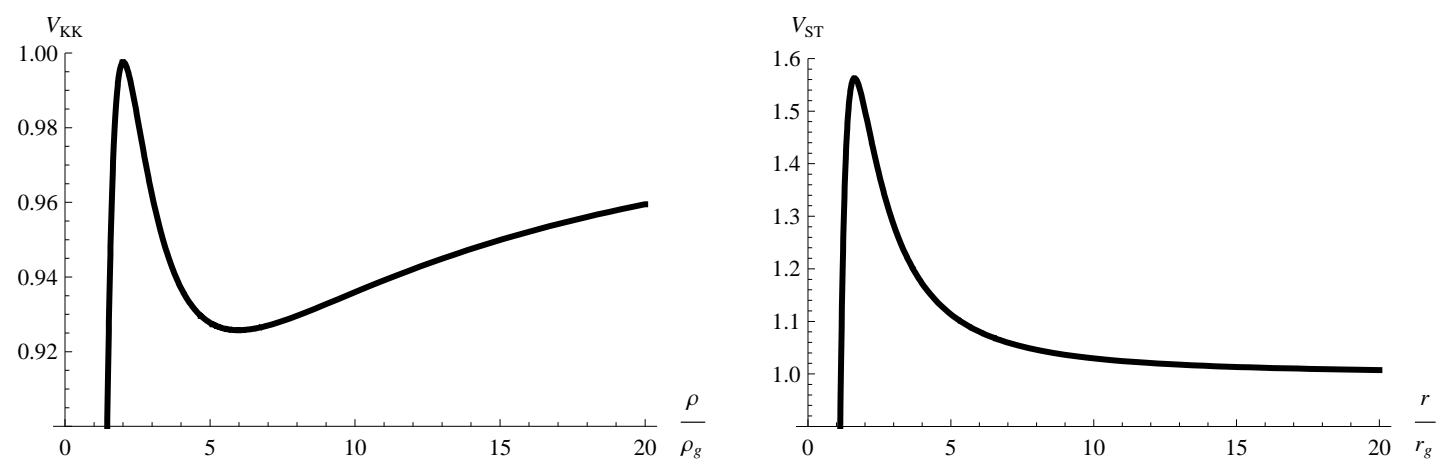

FIG. 1: Effective potentials for a massive test particle moving in the five-dimensional squashed Kaluza-Klein black hole metric (left). We set the parameters as $L=2 \rho_{g}$ and $\rho_{0}=10^{-2} \rho_{g}$ in (17). The same one in the five-dimensional Schwarzschild-Tangherlini black hole metric (right) with the parameters $L_{\mathrm{ST}}=2 r_{g}$ in (19).

In the present paper, we restrict ourselves to a circular motion $\rho=R=$ const. with $p_{\psi}=0$ and $\theta=\pi / 2$. Then we have

$$
u^{\rho}=0, \quad u^{\theta}=0, \quad \text { and } \quad u^{\psi}=0,
$$

where $u^{\mu}=d x^{\mu} / d \tau$ is the five-velocity of the particle. From $V_{\mathrm{KK}}=E^{2}$ and $d V_{\mathrm{KK}} / d \rho=0$, we have

$$
E^{2}=\frac{\left(R-\rho_{g}\right)^{2}\left(2 R+\rho_{0}\right)}{R^{2}\left(2 R-3 \rho_{g}\right)+R \rho_{0}\left(R-2 \rho_{g}\right)}, \quad L^{2}=\frac{\rho_{g} R\left(R+\rho_{0}\right)^{2}}{R\left(2 R-3 \rho_{g}\right)+\rho_{0}\left(R-2 \rho_{g}\right)} .
$$

and

$$
\begin{aligned}
u^{t} & =\sqrt{\frac{R\left(2 R+\rho_{0}\right)}{R\left(2 R-3 \rho_{g}\right)+\rho_{0}\left(R-2 \rho_{g}\right)}}, \\
u^{\phi} & =\sqrt{\frac{\rho_{g}}{R^{2}\left(2 R-3 \rho_{g}\right)+\rho_{0} R\left(R-2 \rho_{g}\right)}} .
\end{aligned}
$$

By using (7), (22) and (23), we obtain Kepler's third law in the squashed Kaluza-Klein black hole spacetime as

$$
T^{2}=\frac{4 \pi^{2}}{G_{4} M} R^{3}\left(1+\frac{1}{2} \frac{\rho_{0}}{R}\right),
$$

where $T$ denotes the orbital period. The second term in the right hand side is the correction by the extra dimension. 


\section{VERIFICATION OF EXTRA DIMENSION BY GEODETIC EFFECT OBSER- VATION}

We regard the rotating axis of the gyroscope carried by a satellite as a spacelike spinvector $S^{\mu}$ parallely transported along a timelike geodesic with the five-velocity $u^{\mu}$. The parallel transporting equation of $S^{\mu}$ in the direction of $u^{\mu}$ is

$$
u^{\mu} \nabla_{\mu} S^{\nu}=0
$$

We impose the orthogonality condition between $u^{\mu}$ and $S^{\mu}$, and normalization condition

$$
u^{\mu} S_{\mu}=0, \quad S^{\mu} S_{\mu}=1
$$

The parallel transporting equation (25) along the circular orbits in the squashed KaluzaKlein spacetime becomes

$$
\begin{aligned}
& \frac{d S^{t}}{d \tau}+\frac{\rho_{g} u^{t}}{2 R\left(R-\rho_{g}\right)} S^{\rho}=0, \\
& \frac{d S^{\rho}}{d \tau}+\frac{\rho_{g}\left(R-\rho_{g}\right) u^{t}}{2 R^{2}\left(R+\rho_{0}\right)} S^{t}-\frac{\left(R-\rho_{g}\right)\left(2 R+\rho_{0}\right) u^{\phi}}{2\left(R+\rho_{0}\right)} S^{\phi}=0, \\
& \frac{d S^{\phi}}{d \tau}+\frac{\left(2 R+\rho_{0}\right) u^{\phi}}{2 R\left(R+\rho_{0}\right)} S^{\rho}=0, \\
& \frac{d S^{\theta}}{d \tau}+\frac{\rho_{0}\left(\rho_{g}+\rho_{0}\right) u^{\phi}}{2\left(R+\rho_{0}\right)^{2}} S^{\psi}=0, \\
& \frac{d S^{\psi}}{d \tau}-\frac{u^{\phi}}{2} S^{\theta}=0 .
\end{aligned}
$$

By using (22) and (23) for the explicit forms of $u^{t}$ and $u^{\phi}$, we obtain the spin-vector $S^{\mu}$ as

$$
\begin{aligned}
S^{t} & =C^{t} \sin (\Omega \tau), \\
S^{\rho} & =C^{\rho} \cos (\Omega \tau), \\
S^{\phi} & =\frac{C^{\phi}}{R} \sin (\Omega \tau), \\
S^{\theta} & =\frac{C^{\theta}}{R} \cos (\tilde{\Omega} \tau), \\
S^{\psi} & =\frac{C^{\psi}}{R} \sin (\tilde{\Omega} \tau),
\end{aligned}
$$

where $\Omega$ and $\tilde{\Omega}$ are given as

$$
\Omega=\frac{\sqrt{\rho_{g}\left(2 R+\rho_{0}\right)}}{2 R\left(R+\rho_{0}\right)},
$$




$$
\tilde{\Omega}=\frac{1}{2\left(R+\rho_{0}\right)} \sqrt{\frac{\rho_{g} \rho_{0}\left(\rho_{g}+\rho_{0}\right)}{R\left[R\left(2 R-3 \rho_{g}\right)+\rho_{0}\left(R-2 \rho_{g}\right)\right]}} .
$$

We have imposed initial condition $S^{t}=S^{\phi}=S^{\psi}=0$ at $\tau=0$, for convenience. Constants $C^{\mu}$ are determined by the orthogonality and the normalization condition (26) as

$$
\begin{aligned}
& C^{t}=C^{\rho} \frac{R+\rho_{0}}{R-\rho_{g}} \sqrt{\frac{\rho_{g} R}{R\left(2 R-3 \rho_{g}\right)+\rho_{0}\left(R-2 \rho_{g}\right)}}, \\
& C^{\phi}=C^{\rho} \sqrt{\frac{R\left(2 R+\rho_{0}\right)}{R\left(2 R-3 \rho_{g}\right)+\rho_{0}\left(R-2 \rho_{g}\right)}}, \\
& C^{\theta}=\sqrt{\frac{R}{R+\rho_{0}}\left(1-\frac{R+\rho_{0}}{R-\rho_{g}}\left(C^{\rho}\right)^{2}\right)}, \\
& C^{\psi}=\sqrt{\frac{R\left(R+\rho_{0}\right)}{\rho_{0}\left(\rho_{g}+\rho_{0}\right)}\left(1-\frac{R+\rho_{0}}{R-\rho_{g}}\left(C^{\rho}\right)^{2}\right)} .
\end{aligned}
$$

Since we consider the situation such as $\rho_{0} \ll \rho_{g} \ll R$, we have $\tilde{\Omega} \ll \Omega$. Then, we can regard $S^{\theta}$ and $S^{\psi}$ are almost constant for some orbital periods. Further, we have $C^{t} \ll C^{\phi} \simeq C^{\rho}$.

During one orbital period, $\phi$ goes from 0 to $2 \pi$ and a proper time $\tau$ goes from 0 to $\tau_{p}:=2 \pi / u^{\phi}$, the geodetic precession angle $\Delta \Theta$ is given as

$$
\Delta \Theta=\frac{R S^{\phi}\left(\tau_{p}\right)}{S^{\rho}(0)}=\left|\Omega \tau_{p}-2 \pi\right|=\left|2 \pi\left(\frac{\Omega}{u^{\phi}}-1\right)\right| .
$$

Substituting (23) and (37) into (43), we obtain the geodetic precession angle in a weak-field limit as

$$
\Delta \Theta=\Delta \Theta_{4 \mathrm{D}}(1+\delta)+O\left(R^{-2}\right)
$$

where

$$
\Delta \Theta_{4 \mathrm{D}}=\frac{3 \pi G_{4} M}{R}
$$

is the predicted value by the four-dimensional Einstein theory and

$$
\delta=\frac{\rho_{0}\left(\rho_{0}+\rho_{g}\right)}{6\left(G_{4} M\right)^{2}}=\frac{1}{6}\left(\frac{r_{\infty}}{2 G_{4} M}\right)^{2}
$$

is the higher-dimensional correction. For $r_{\infty} \rightarrow 0$, equivalently $\rho_{0} \rightarrow 0$, the correction $\delta$ vanishes as expected. If the size of extra dimension is $r_{\infty} \simeq 0.1 \mathrm{~mm}$, we can estimate that $\delta \simeq 10^{-5}$ for the Earth. 


\section{DISCUSSIONS}

In this paper, we have considered the precession of a gyroscope in a circular orbit around a spherical compact object. We have assumed that the five-dimensional squashed KaluzaKlein black hole solution describes the geometry around the compact object. We have solved the parallel transporting equation of a spin-vector along the circular timelike geodesic in the squashed Kaluza-Klein black hole spacetime, and derived the geodetic precession angle with the higher-dimensional correction. We have shown that the correction to the general relativity is in proportion to the square of (size of extra dimension)/(gravitational radius of central object). If the size of extra dimension is order of $0.1 \mathrm{~mm}$, the correction is order of $10^{-5}$ for the Earth.

In the four-dimensional general relativity, the gravitational field in vacuum with spherical symmetry is uniquely described by the Schwarzschild metric. However, in the higherdimensional spacetime with Kaluza-Klein structure, even if we impose an asymptotic flatness in four-dimensional section, the metric is not determined uniquely. For example, the direct

product of four-dimensional Schwarzschild spacetime with a small $\mathrm{S}^{1}$ is a possible metric to describe the geometry around the Earth. In this case, no higher-dimensional correction appears without a momentum of gyroscope in the extra direction. In contrast, it is interesting that the correction exists even if the gyroscope moves along four-dimensional spacetime in the squashed Kaluza-Klein geometry. If a precise experiment of gyroscope precession in the Earth orbit agrees the expected value of general relativity, it requires a rigorous upper limit of the size of extra dimension, or it excludes the squashed Kaluza-Klein metric for describing the geometry around the Earth.

\section{Acknowledgments}

We would like to thank M. Kimura, T. Nakagawa, K. Nakao and S. Tomizawa for useful discussions. This work is supported by the Grant-in-Aid for Scientific Research No.19540305. 
[1] R. Emparan and H. S. Reall, "Black Holes in Higher Dimensions", Living Rev. Relativity 11, 6 (2008): http://www.livingreviews.org/lrr-2008-6.

[2] T.Banks and W.Fischler, arXiv:hep-th/9906038

[3] S. Dimopoulos and G. Landsberg, Phys. Rev. Lett. 87, 161602 (2001).

[4] S.B. Giddings and S. Thomas, Phys. Rev. D 65, 056010 (2002).

[5] N. Arkani-Hamed, S. Dimopoulos, and G. R. Dvali, Phys. Rev. D 59, 086004 (1999).

[6] P. C. Argyres, S. Dimopoulos, and J. March-Russell, Phys. Lett. B 441, 96 (1998).

[7] J.L. Feng and A.D. Shapere, Phys. Rev. Lett. 88, 021303 (2002).

[8] L.A. Anchordoqui, J.L. Feng, H. Goldberg, and A.D. Shapere, Phys. Rev. D 65, 124027 (2002).

[9] S. S. Seahra, C. Clarkson and R. Maartens, Phys. Rev. Lett. 94, 121302 (2005).

[10] W. de Sitter, Mon. Not. Royal Astron. Soc. 77, 155 (1916).

[11] J. Lense and H. Thirring, Phys. Zeit. 19, 156 (1918).

[12] Gravity Probe B web site: http://einstein.stanford.edu/

[13] P.H. Lim and P.S. Wesson, Astrophys. J. 397, L91 (1992).

[14] D. Kalligas, P.S. Wesson, and C.W.F. Everitt, Astrophys. J. 439, 548 (1995).

[15] P.H. Lim, J.M. Overduin, and P.S. Wesson, J. Math. Phys. 36, 6907 (1995).

[16] H. Liu and P.S. Wesson, Class. Quant. Grav. 14, 1651 (1997).

[17] J.M. Overduin, Phys. Rev. D 62, 102001 (2000).

[18] H. Liu and J.M. Overduin, Astrophys. J. 538, 386 (2000).

[19] H. Liu and P.S. Wesson, Class. Quant. Grav. 13, 2311 (1996).

[20] C. G. Böhmer, T. Harko, and F. S. N. Lobo, Class. Quant. Grav. 25, 045015 (2008).

[21] S. Jalalzadeh, M. Mehrnia, and H. R. Sepangi, Class. Quant. Grav. 26, 155007 (2009).

[22] S. S. Seahra, Phys. Rev. D 65, 124004 (2002).

[23] P. Dobiasch and D. Maison, Gen. Rel. Grav. 14, 231 (1982).

[24] G.W. Gibbons and D.L. Wiltshire, Ann. Phys. 167, 201 (1986).

[25] D. Rasheed, Nucl. Phys. B 454, 379 (1995).

[26] H. Ishihara and K. Matsuno, Prog. Theor. Phys. 116, 417 (2006).

[27] T. Wang, Nucl. Phys. B 756, 86 (2006). 
[28] D. Gaiotto, A. Strominger, and X. Yin, JHEP 02, 024 (2006).

[29] H. Ishihara, M. Kimura, K. Matsuno, and S. Tomizawa, Class. Quant. Grav. 23, 6919 (2006).

[30] K. Matsuno, H. Ishihara, T. Nakagawa, and S. Tomizawa, Phys. Rev. D 78, 064016 (2008).

[31] J. P. Gauntlett, J. B. Gutowski, C. M. Hull, S. Pakis, and H. S. Reall, Class. Quant. Grav. 20, 4587 (2003).

[32] T. Nakagawa, H. Ishihara, K. Matsuno, and S. Tomizawa, Phys. Rev. D 77, 044040 (2008).

[33] S. Tomizawa, H. Ishihara, K. Matsuno, and T. Nakagawa, Prog. Theor. Phys. 121, 823 (2009).

[34] S. Tomizawa and A. Ishibashi, Class. Quant. Grav. 25, 245007 (2008).

[35] C. Stelea, K. Schleich, and D. Witt, Phys. Rev. D 78, 124006 (2008).

[36] S. Tomizawa, Y. Yasui, and Y. Morisawa, Class. Quant. Grav. 26, 145006 (2009).

[37] D. V. Gal'tsov and N. G. Scherbluk, Phys. Rev. D 79, 064020 (2009).

[38] Y. Brihaye and E. Radu, Phys. Lett. B 641, 212 (2006).

[39] S.S. Yazadjiev, Phys. Rev. D 74, 024022 (2006).

[40] M. Allahverdizadeh and K. Matsuno, arXiv:0908.2484 [hep-th].

[41] Y. Kurita and H. Ishihara, Class. Quant. Grav. 24, 4525 (2007).

[42] Y. Kurita and H. Ishihara, Class. Quant. Grav. 25, 085006 (2008).

[43] F.R. Tangherlini, Nuovo Cim. 27, 636 (1963).

[44] V. Frolov and D. Stojkovic, Phys. Rev. D 68, 064011 (2003). 